\title{
New Performance Class
}

\section{Dear Reader,}

The ZF ProAI self-driving system, which is based on the Nvidia Drive PX 2 AI car computer, is the new star presented by ZF Friedrichshafen AG at this year's CES in Las Vegas. In addition to representing a transmission of a new generation with optimised driving power, the system offers integrated computing capacity of a new dimension. Having recently acquired additional know-how in the area of active and passive safety engineering by purchasing TRW, the ZF Group has turned its attention to the vast potential offered by supercomputers and artificial intelligence (AI) as a means of dramatically accelerating the development of driverless vehicle technology.

The appearance of ZF in Las Vegas is symbolic of the change that is occurring on the automobile and mobility market. Although a lot has already been written about this change, many questions remain unanswered and the discussions currently taking place as the entire industry attempts to reorient and reinvent itself remain highly exciting.

Along with the emergence of brand new roles, we can expect to see both winners and losers. We need only consider the remark made by one former electronics executive in the context of an interview with ATZelektronik to the effect that his division had been degraded to the status of a mere service provider to engine developers when it should actually have been the other way around. While many market observers continue to regard the contribution of software, IT and electronics specialists as greatly undervalued, the recent introduction of the executive identifier Chief Digital Officer (CDO) indicates an appropriate response at executive management level. However, experts continue to admonish the industry to make sure the CDOs are the real designers and not merely the implementers.
What is the value of enabling technologies such as high-performance semiconductors? As a matter of fact, the value is very high - although it doesn't necessarily translate into commensurate market recognition. $\mathrm{ZF}$ is one of the few firms that have taken a different approach in the area of marketing/communications. Nvidia has also caught on to the importance of marketing sophistication, as evidenced by the thespian skills it displayed in front of 6000 spectators at the CES.

The CES has become one of the most important performance shows for the automobile industry. German visitors in particular often find themselves asking whether there is a comparably important and impressive exhibition back at home. The answer, unfortunately, is no. While it is too late to compete with the established CES, it would be worth considering ways of improving venues in Germany that are conceived to introduce future technologies - for instance, by making them more accessible to a broader range of interested individuals and stakeholders. We could start by avoiding overly cryptic terms. However, one acronym will continue to make its mark throughout the industry: AI for artificial intelligence.

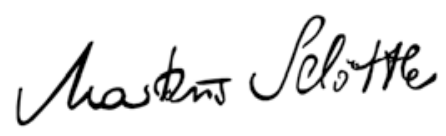

Markus Schöttle Deputy Editor in Chief

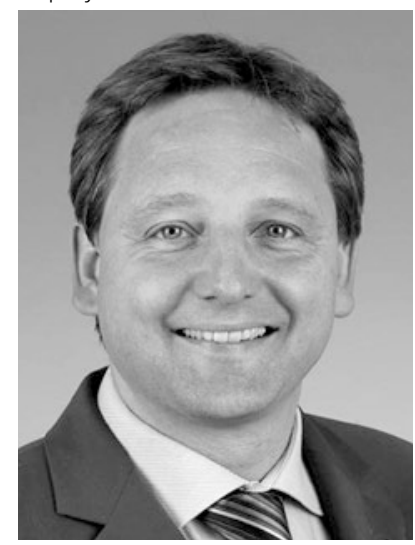

